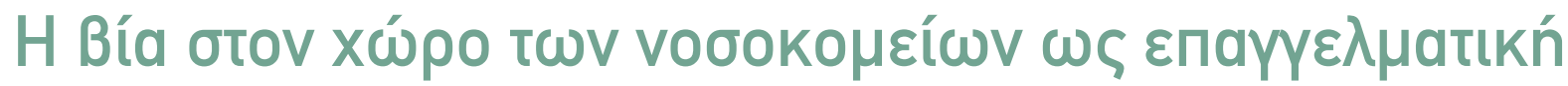

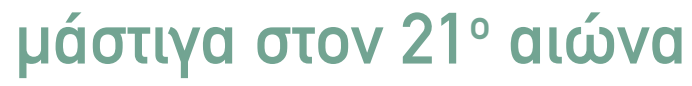

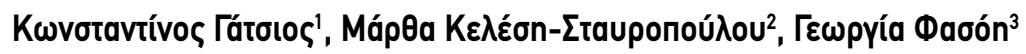

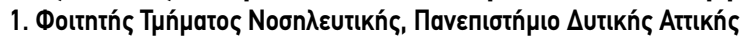

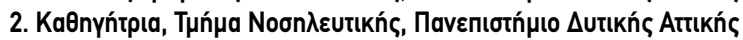

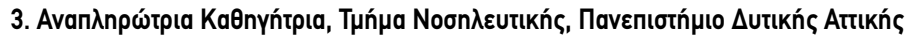

\begin{abstract}
ПЕРIАHШH

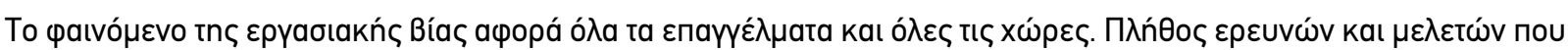

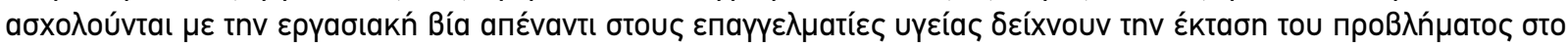

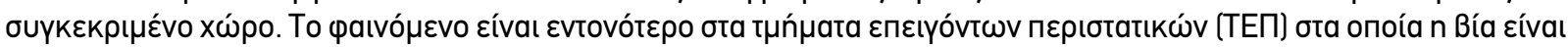

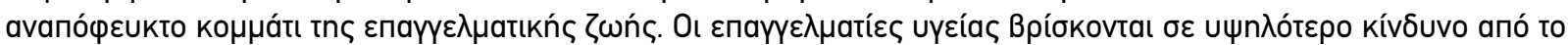

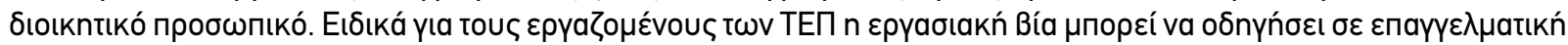

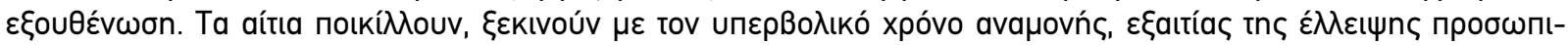

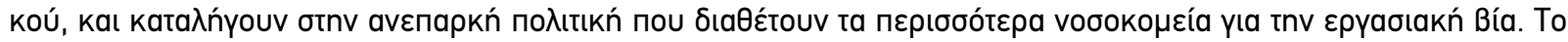

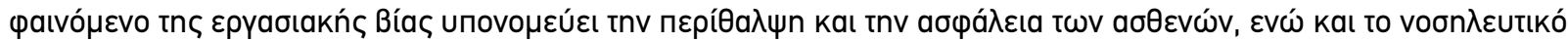

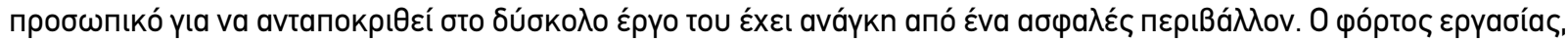

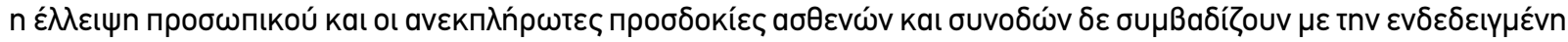

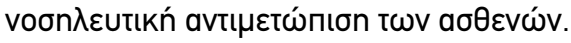

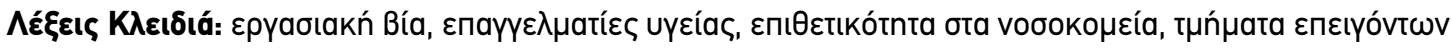

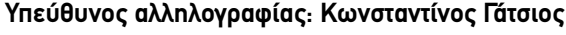
e-mail: gatsioskostas96@gmail.com

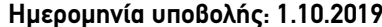

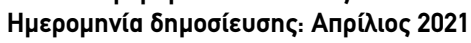

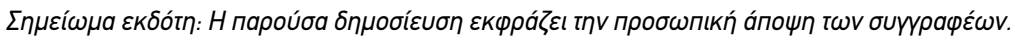

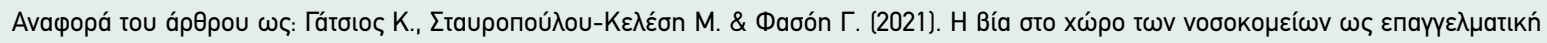

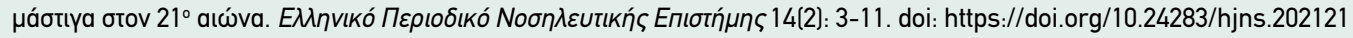




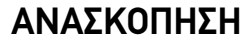

\section{BAEIKA EHMEIA:}

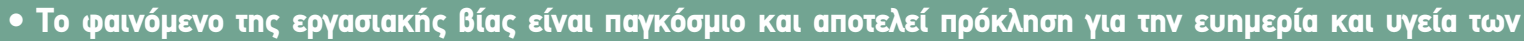

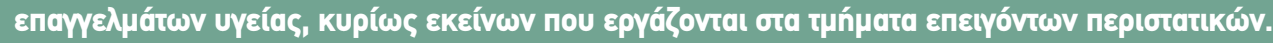

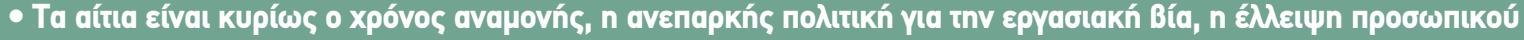

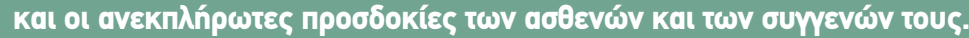

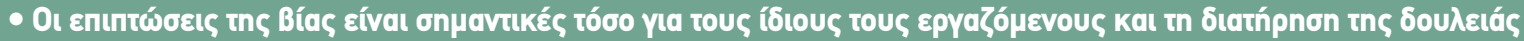

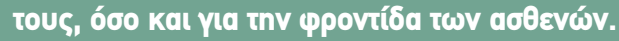

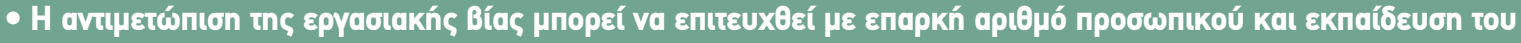

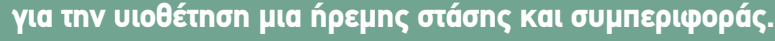

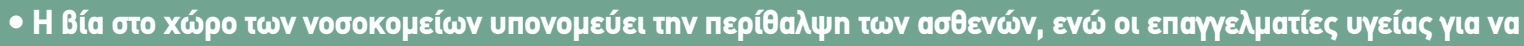

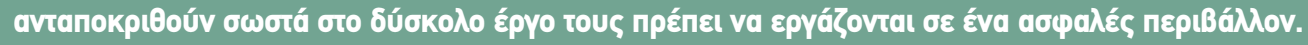

\section{EIIAГRГH}

$\mathrm{T}$ o paivó

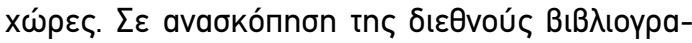

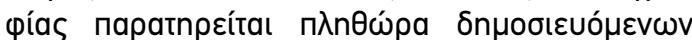

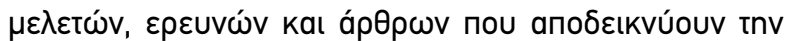

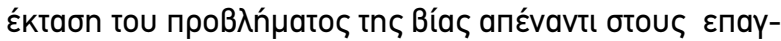

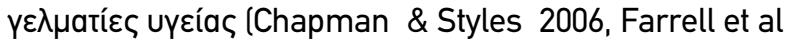

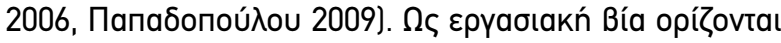

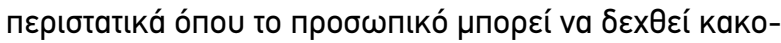

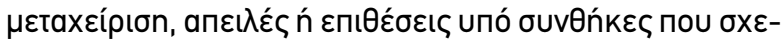

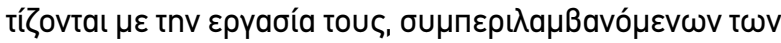

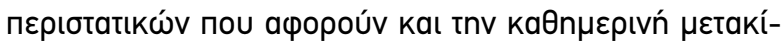

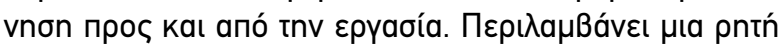

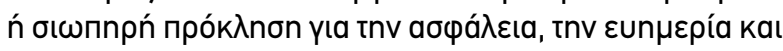

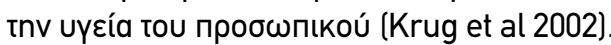

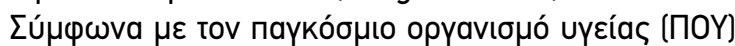

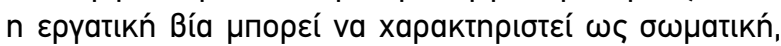

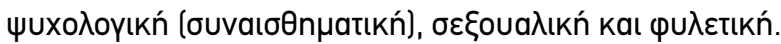

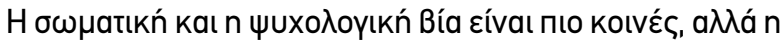

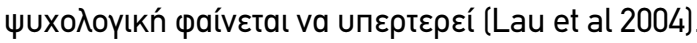

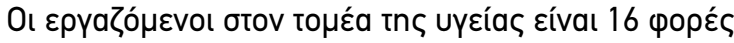

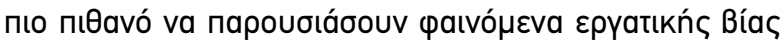

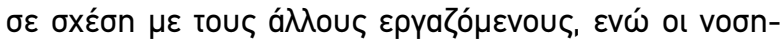

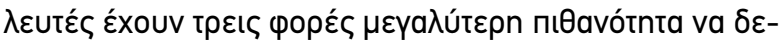

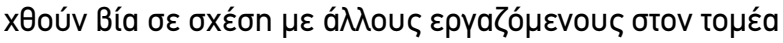

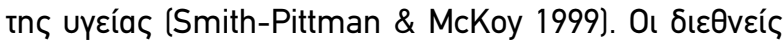

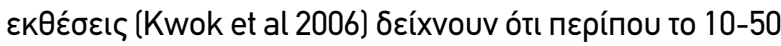

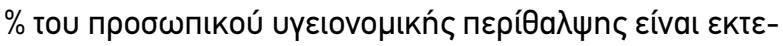

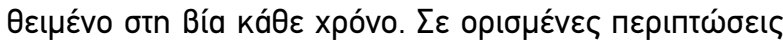

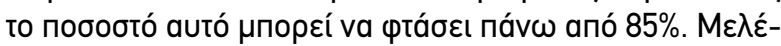

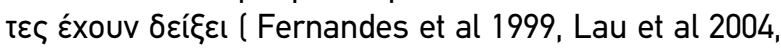
Taylor \& Rew 2011, Gillespie et al 2013) ótı бто xẃpo

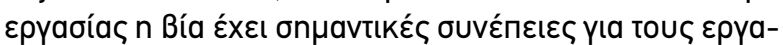

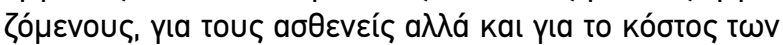

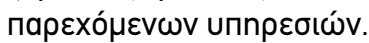

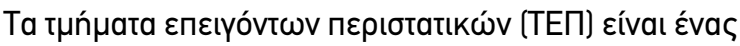

anó touৎ to

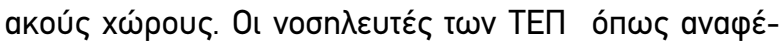
pouv or Shields \& Wilkins (2009), Pich et al (2011), Pich

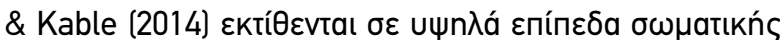

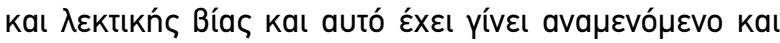

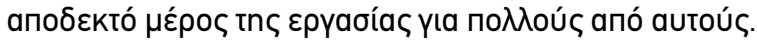

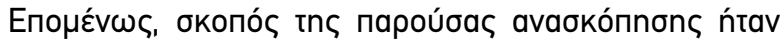

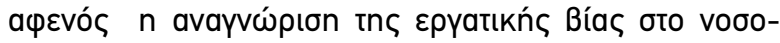

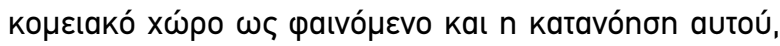

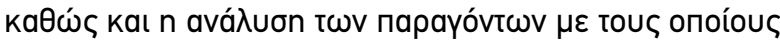

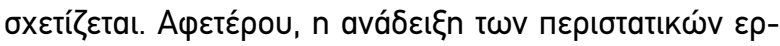

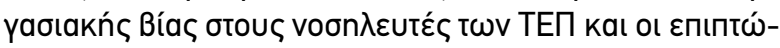

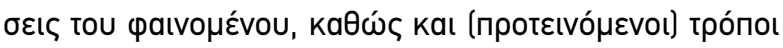

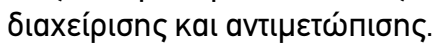

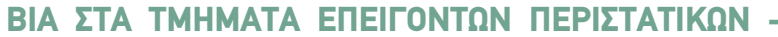 AITIA KAI IAPATONTE $\Sigma$}

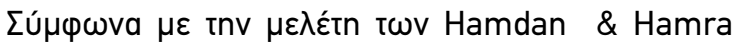

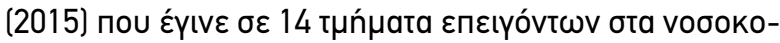

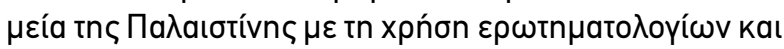

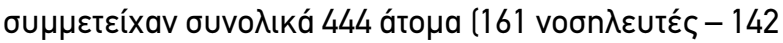

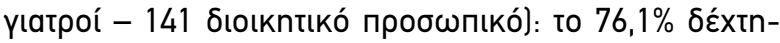

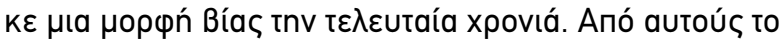

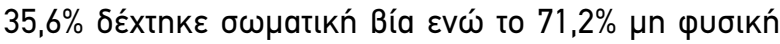

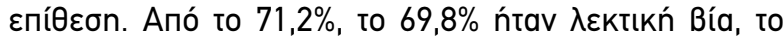

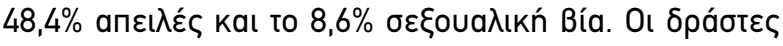

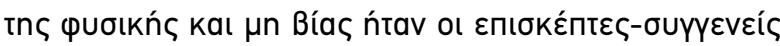

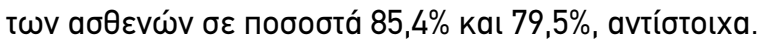

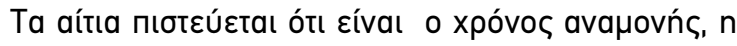

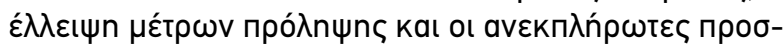

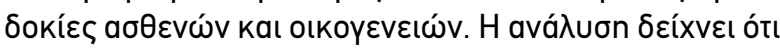

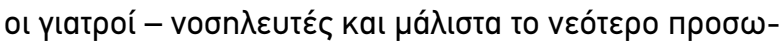

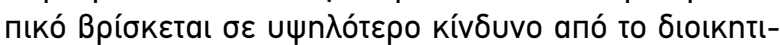

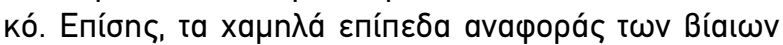

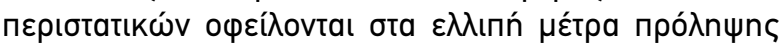

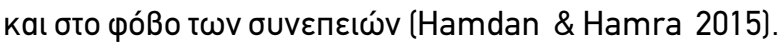




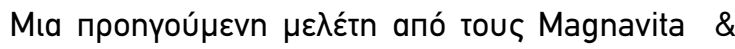

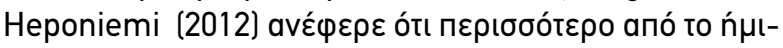

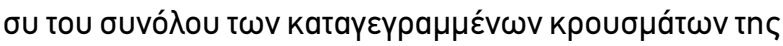

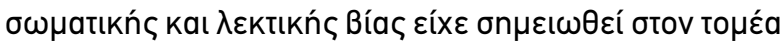

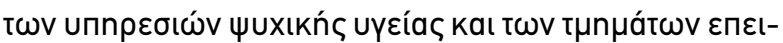

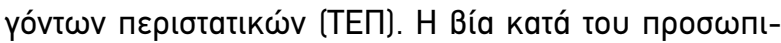

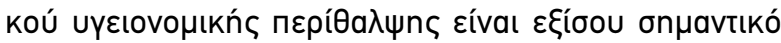

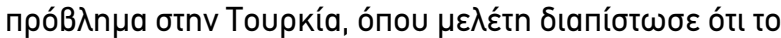

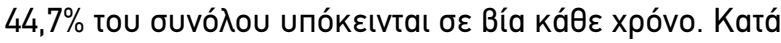

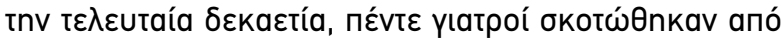

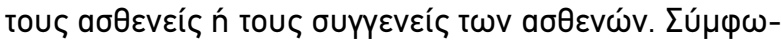

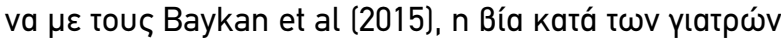
ouvavtátaı nı ouxvá ota TEП tnc xẃpac.

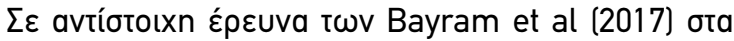

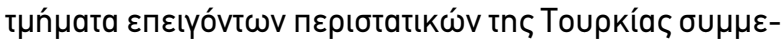

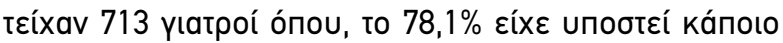

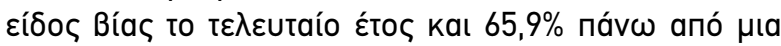

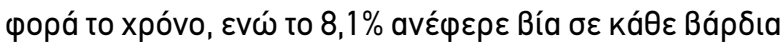

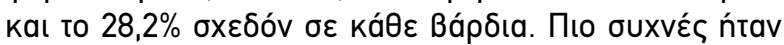

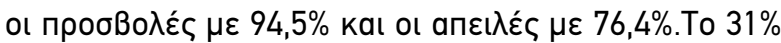

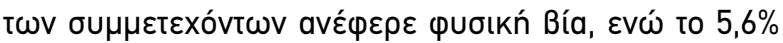

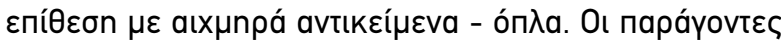

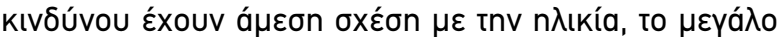

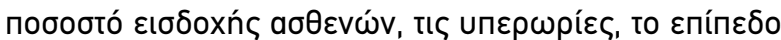

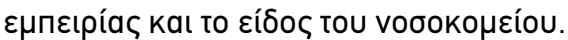

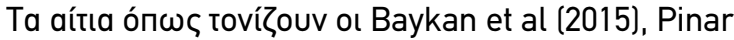

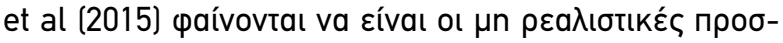

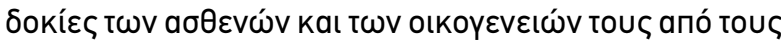

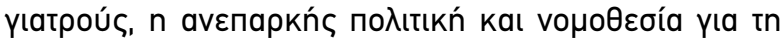

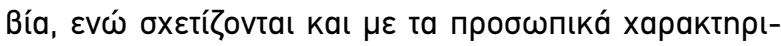

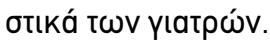

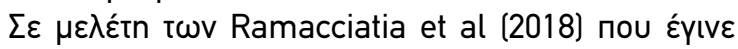

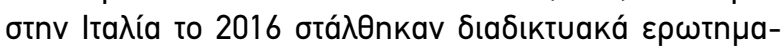

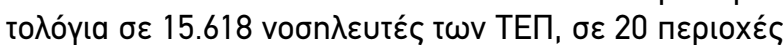

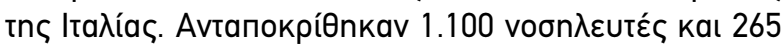

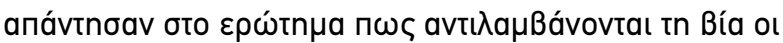

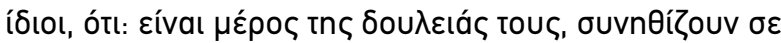

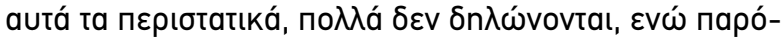

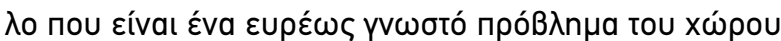

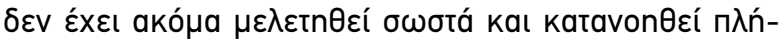

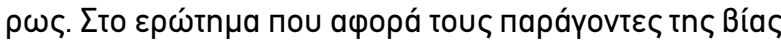

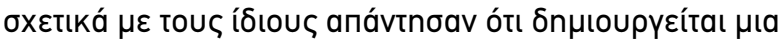

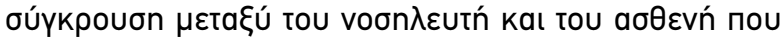

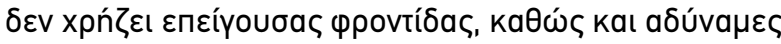

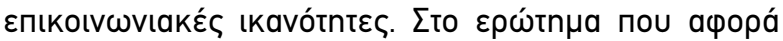

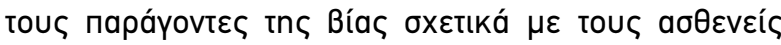

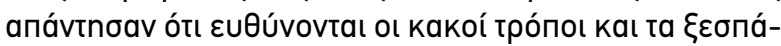

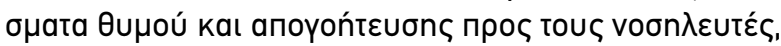
kaӨ

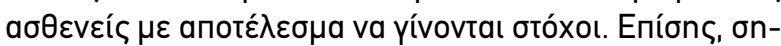

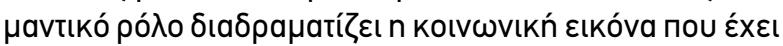

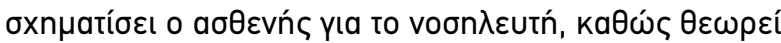

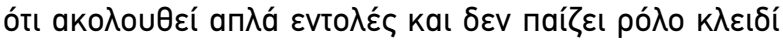

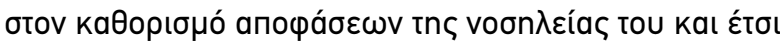

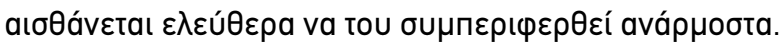

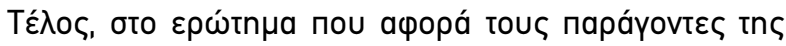

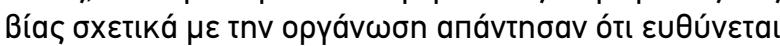

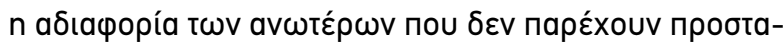

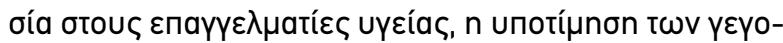

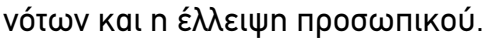

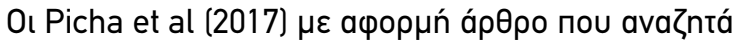

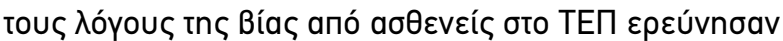

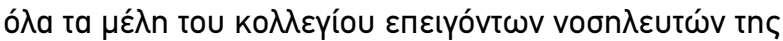

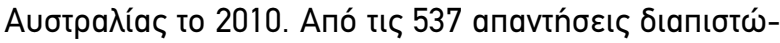

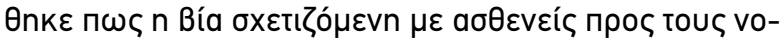

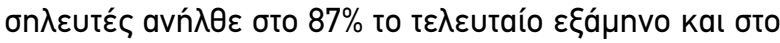

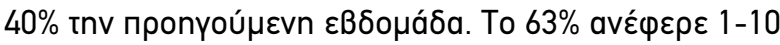

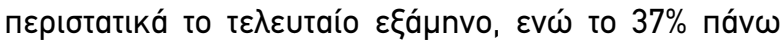

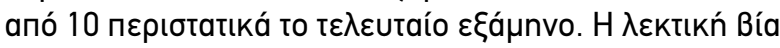

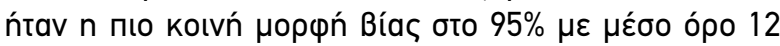

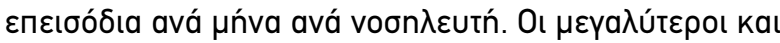

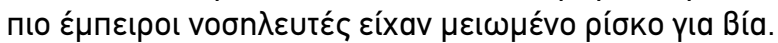

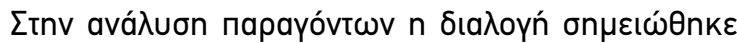

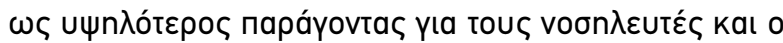

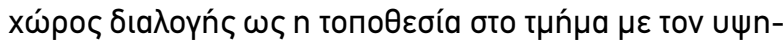

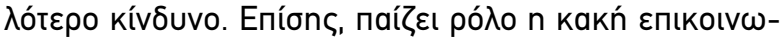

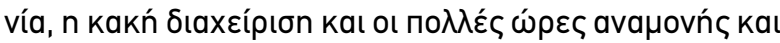

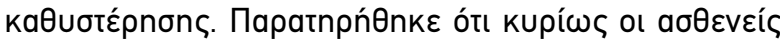

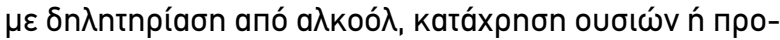

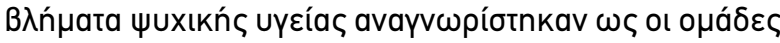
$\mu \varepsilon$ to $\mu \varepsilon \gamma a \lambda u ́ t \varepsilon p o$ kívouvo yıa xpńon Bíac. To $\mu \varepsilon \gamma a \lambda u ́-$

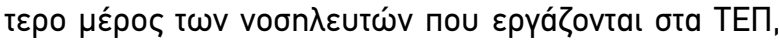

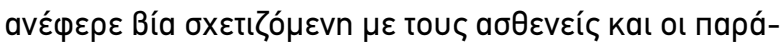

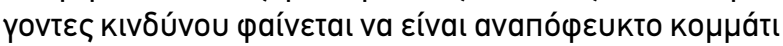

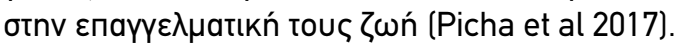

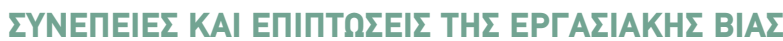

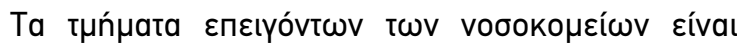

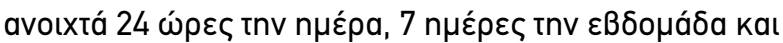

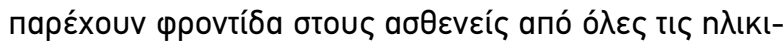

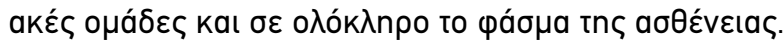

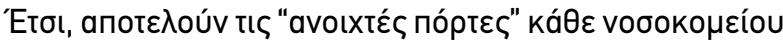

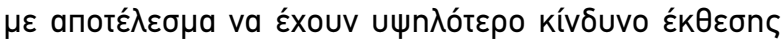

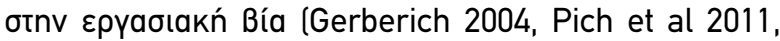

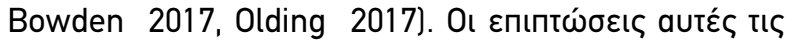

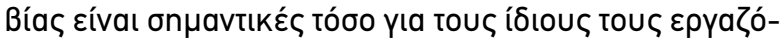

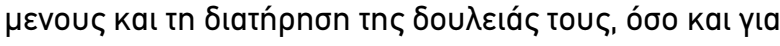
tnv $\varphi \rho o v t i ́ \delta a ~ t \omega v a \sigma \theta \varepsilon v \omega ́ v$.

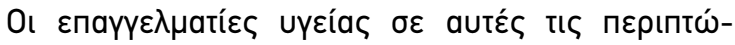

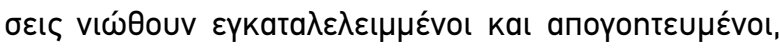

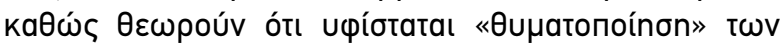

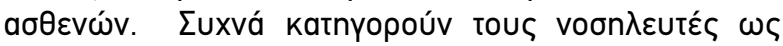

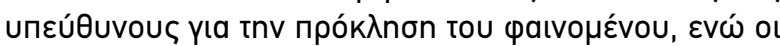

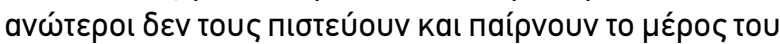

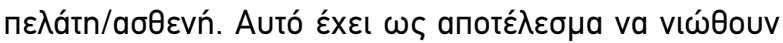

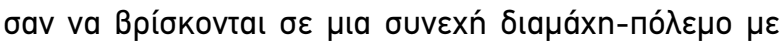




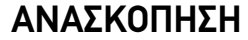

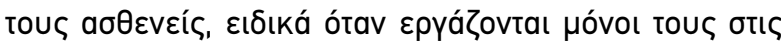

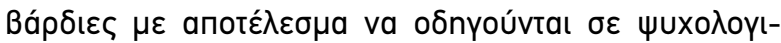

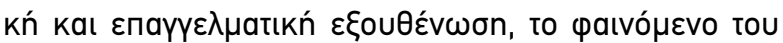
«burnout» (Ramacciatia et al 2018).

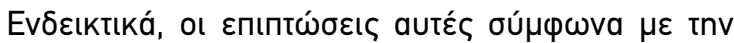

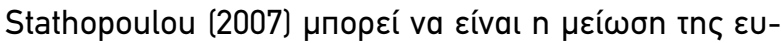

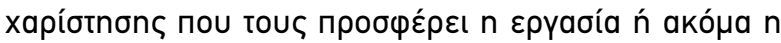

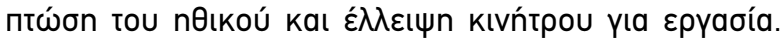

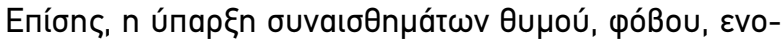

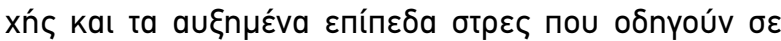

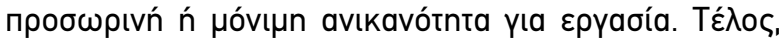

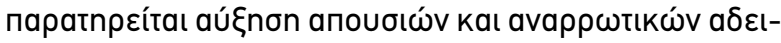
$\omega$ w kat $\mu \varepsilon i ́ \omega \sigma$ tn

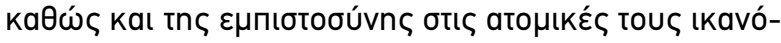
tntє५.

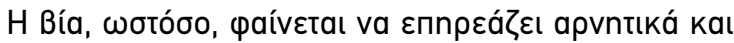

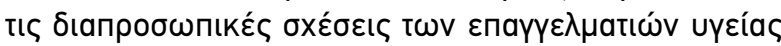

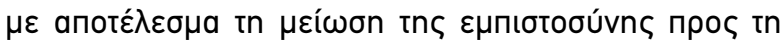

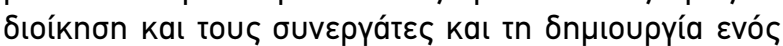

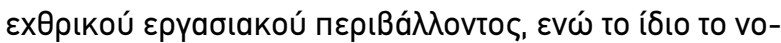

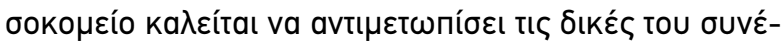

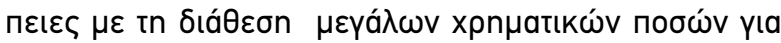

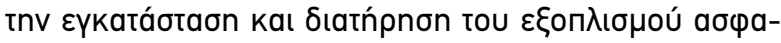
$\lambda \varepsilon$ Éas (Claravall 1996).

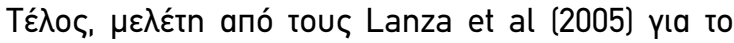

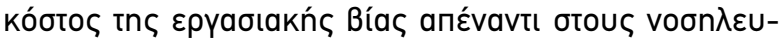

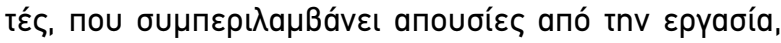

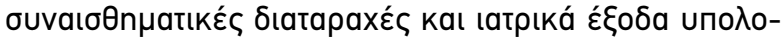

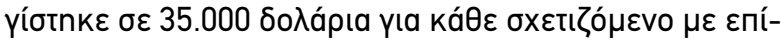

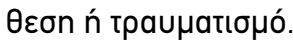

\section{A}

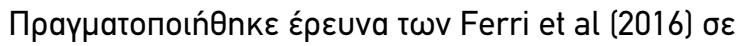

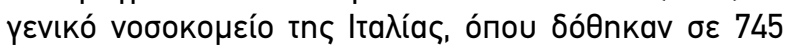

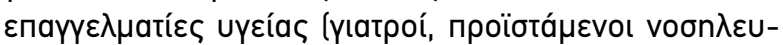

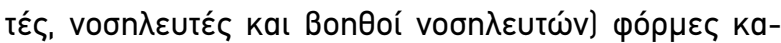

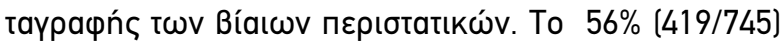

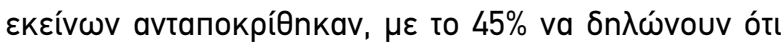

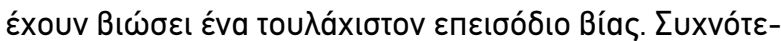

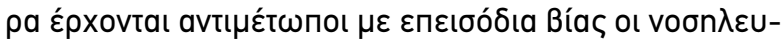

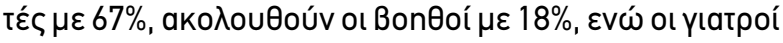

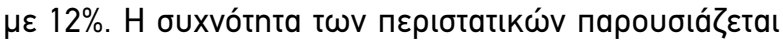

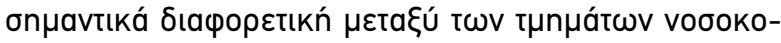

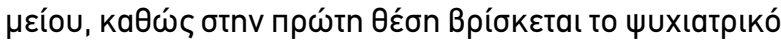

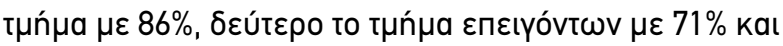

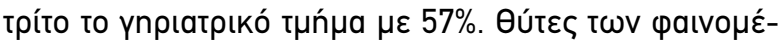

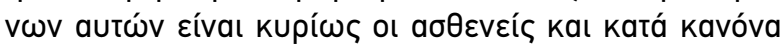

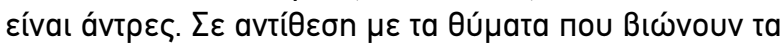

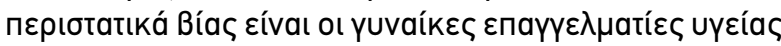

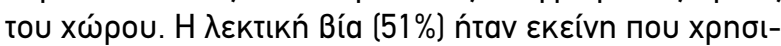

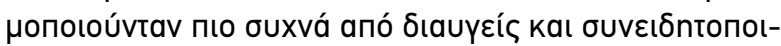

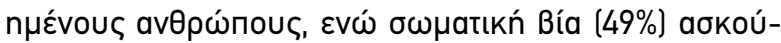

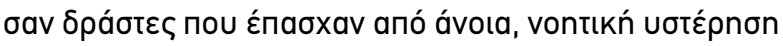

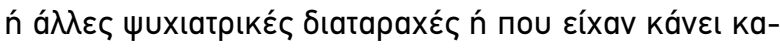

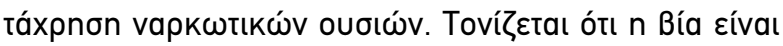

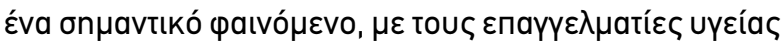

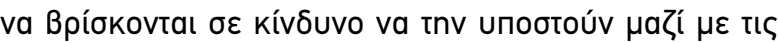

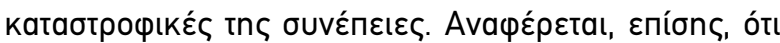

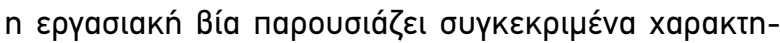

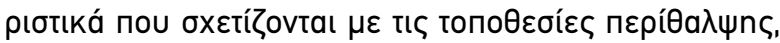

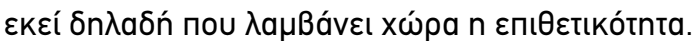

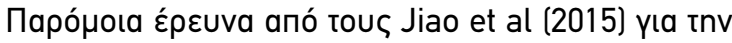

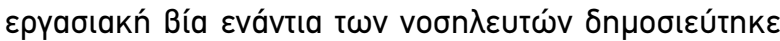

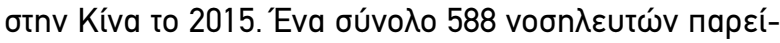

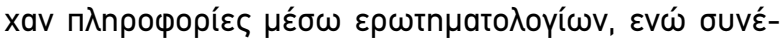

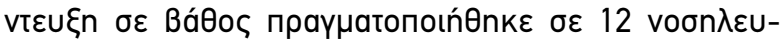

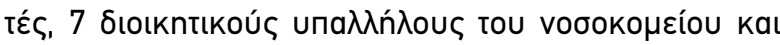

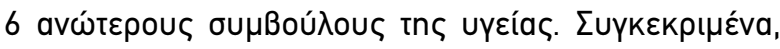

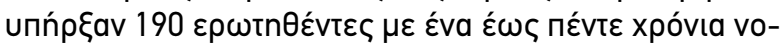

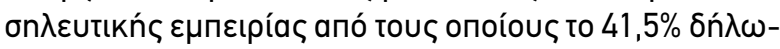

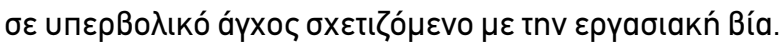

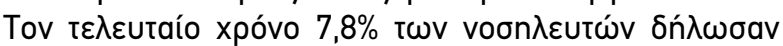

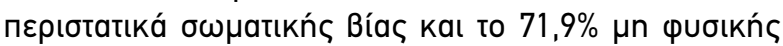

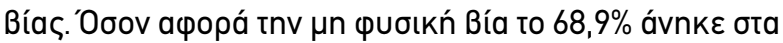

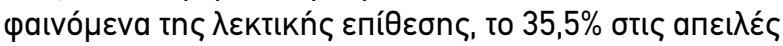

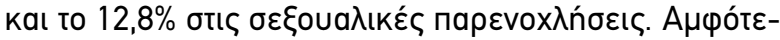

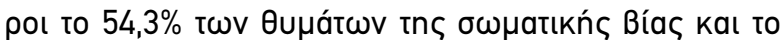

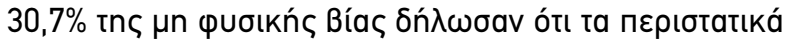

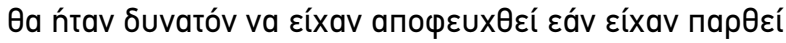

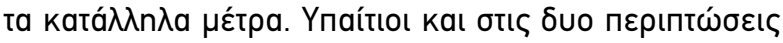

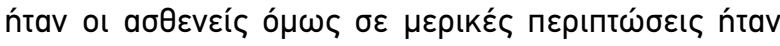

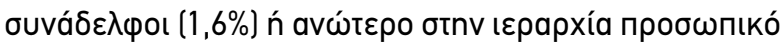

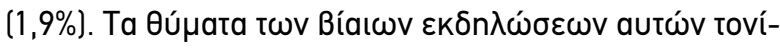

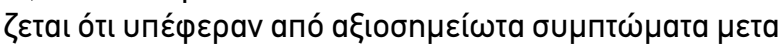

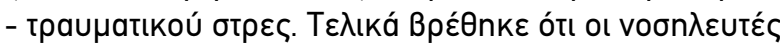

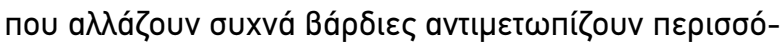

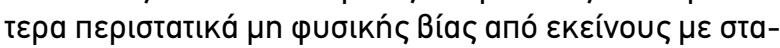

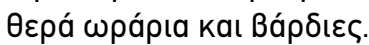

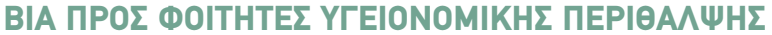

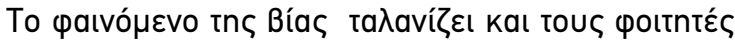

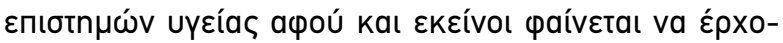

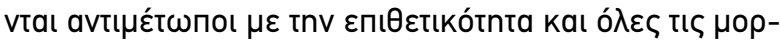

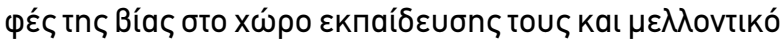

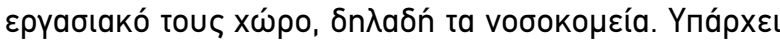

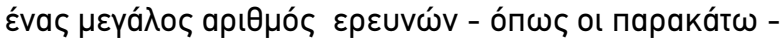

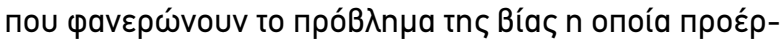

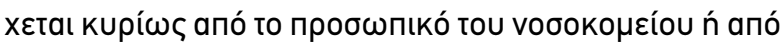

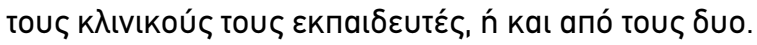

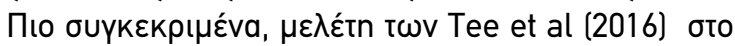

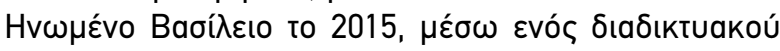

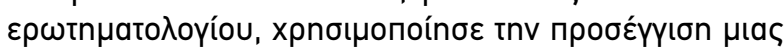

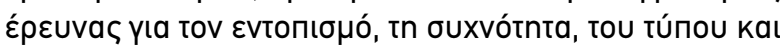

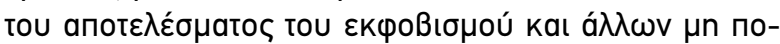

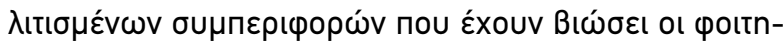

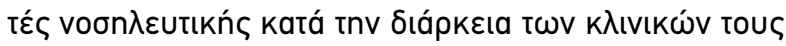




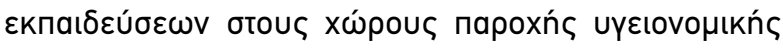

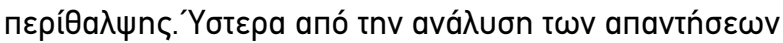

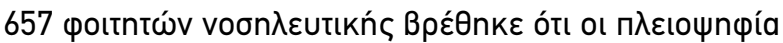

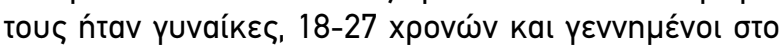

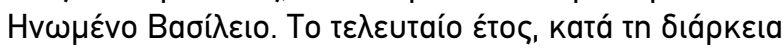

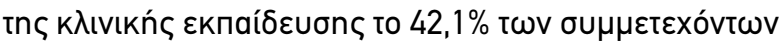

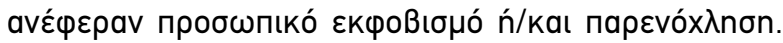

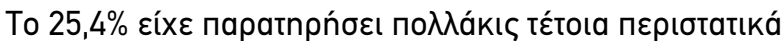

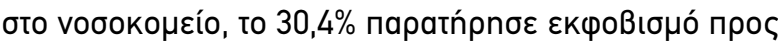

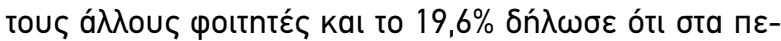

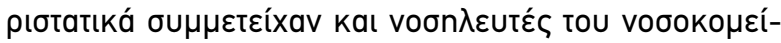

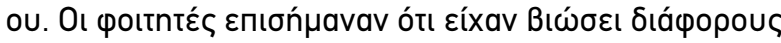

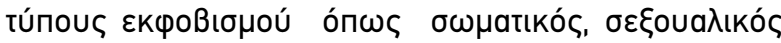

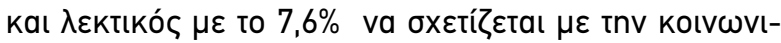

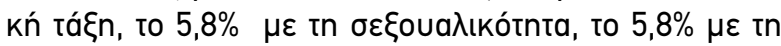

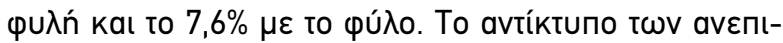

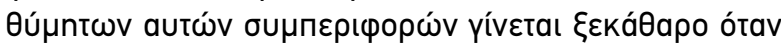

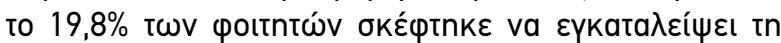

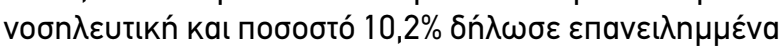

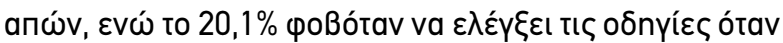

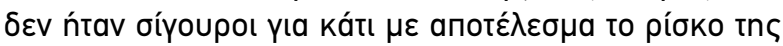

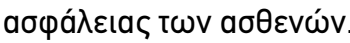

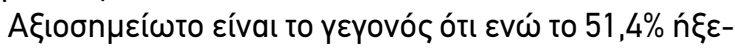

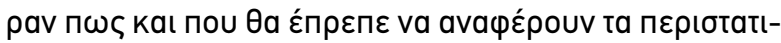

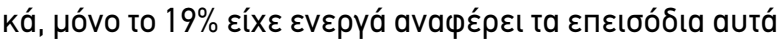

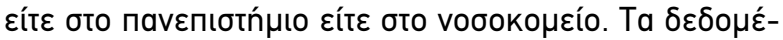

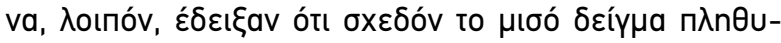

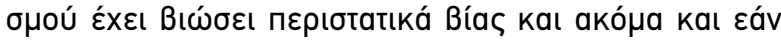

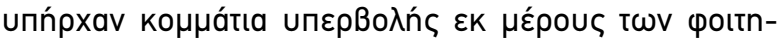

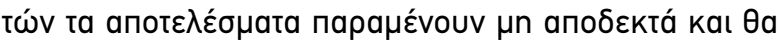

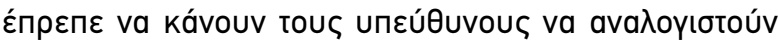

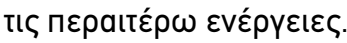

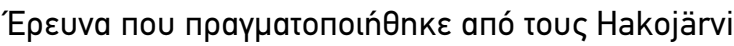

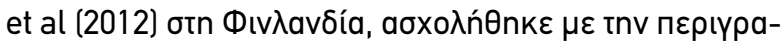

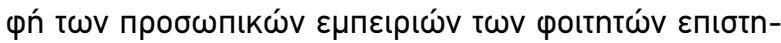

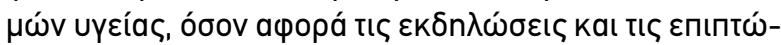

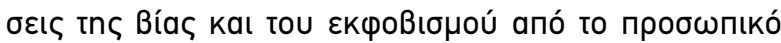

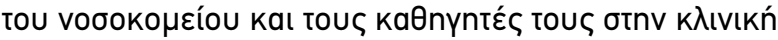

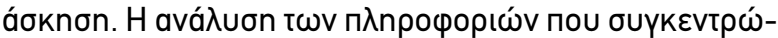

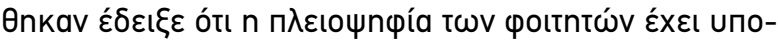

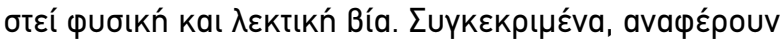

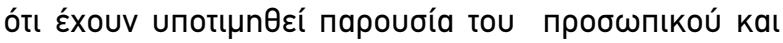

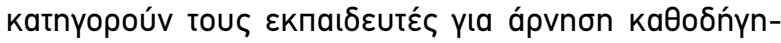

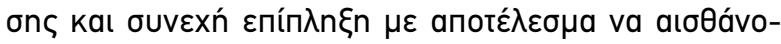

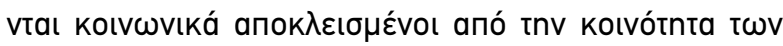

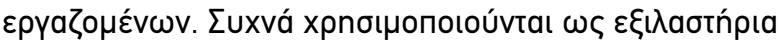

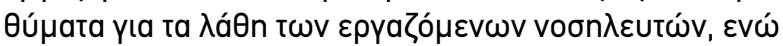

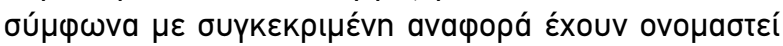

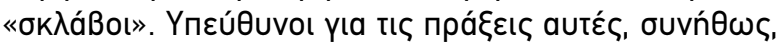

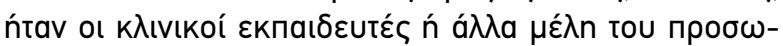

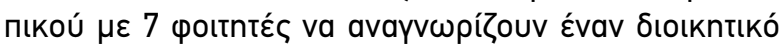

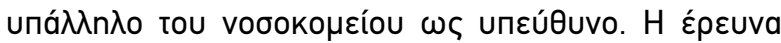

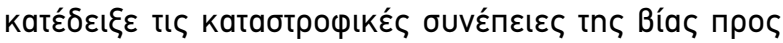

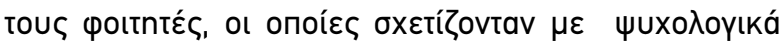

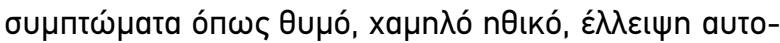

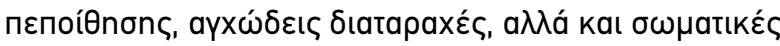

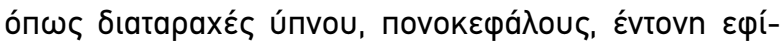

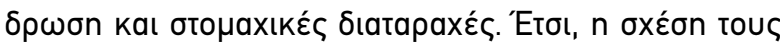

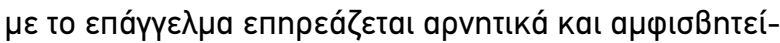

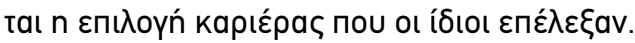

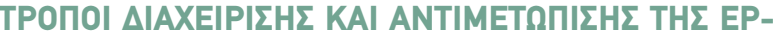 ГA $\Sigma \| A K H \Sigma$ BIA $\Sigma$}

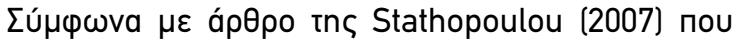

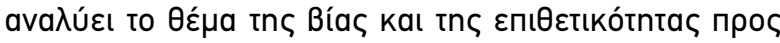

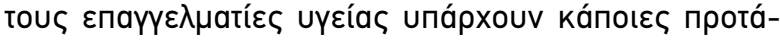

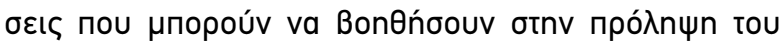

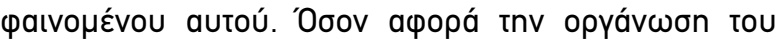

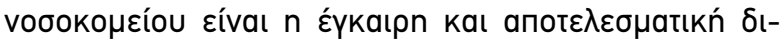

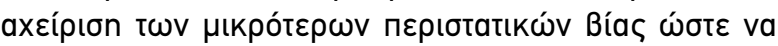

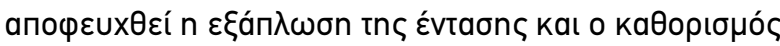

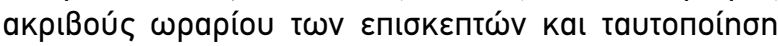

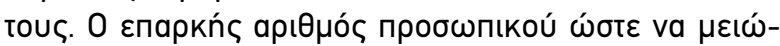

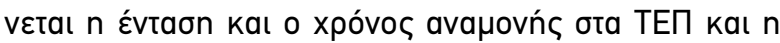

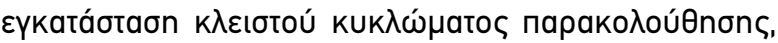

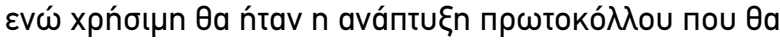

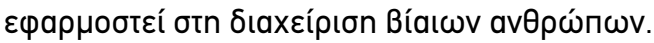

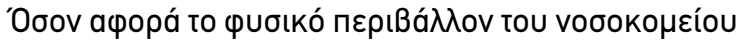

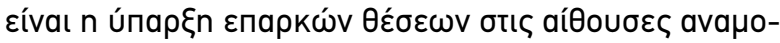

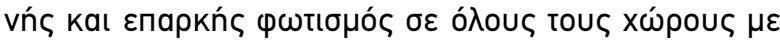

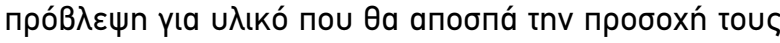

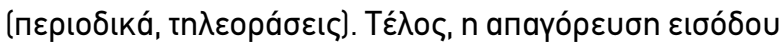

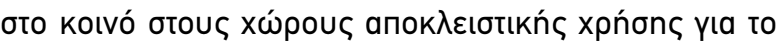

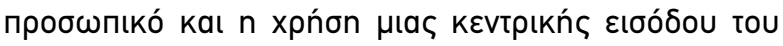

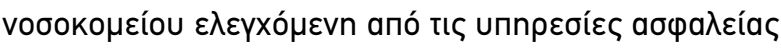

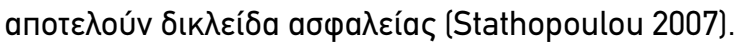

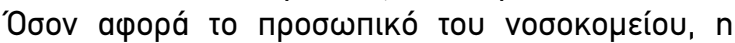

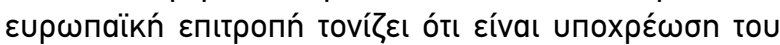

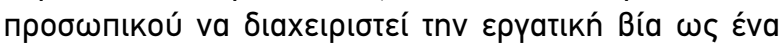
napáyovta kívouvou (American College of Emergency

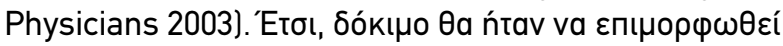

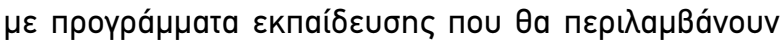

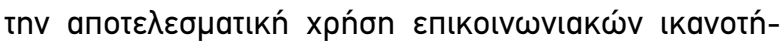

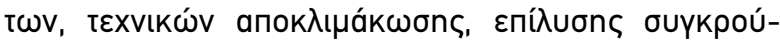

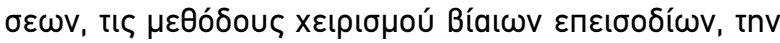

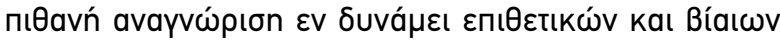

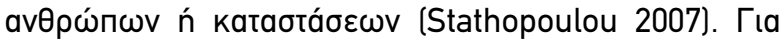

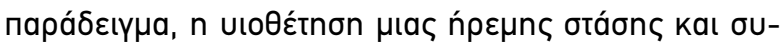

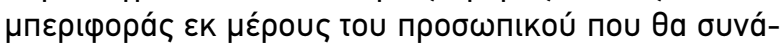

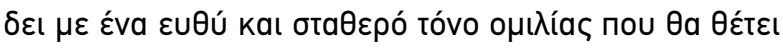

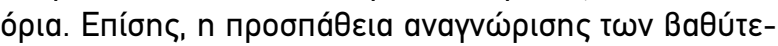

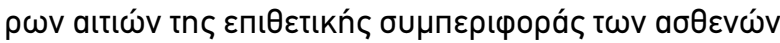

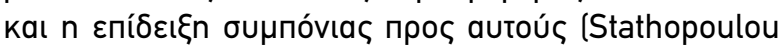
2007).

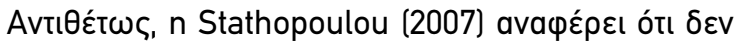

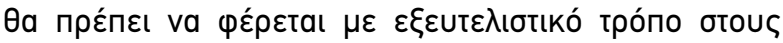




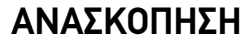

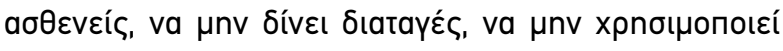

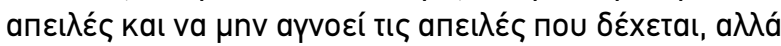

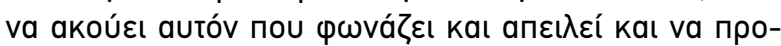

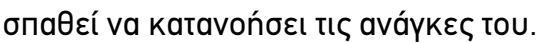

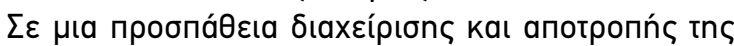

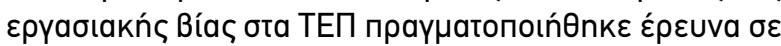

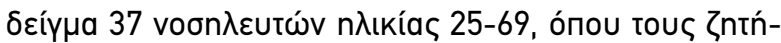

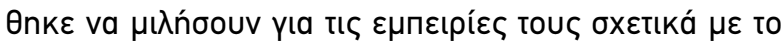

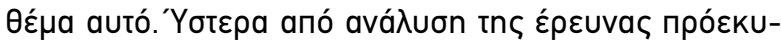

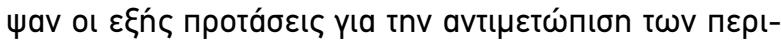

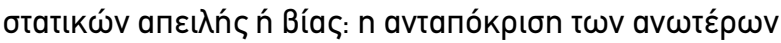

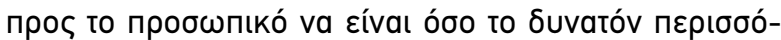

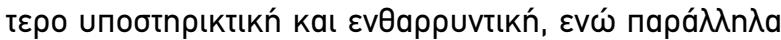

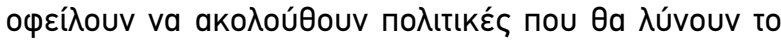

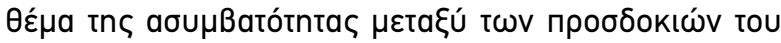

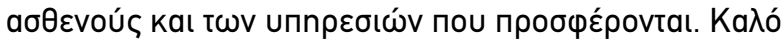

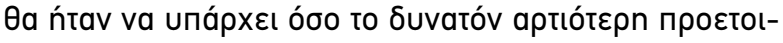

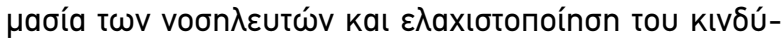

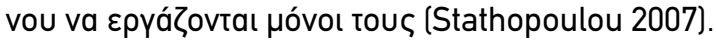

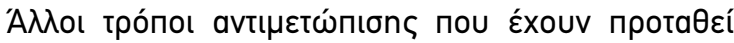

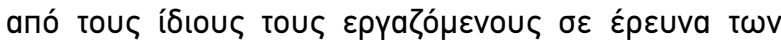

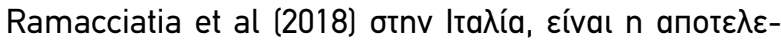

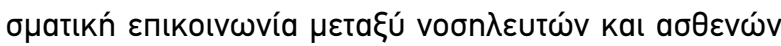

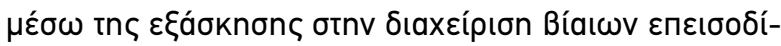

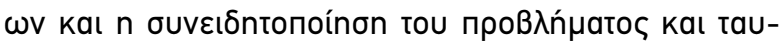

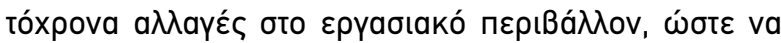

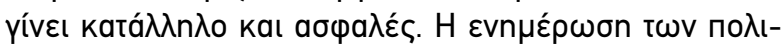

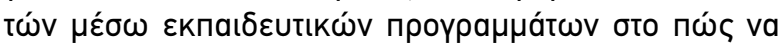

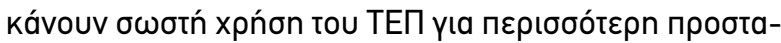

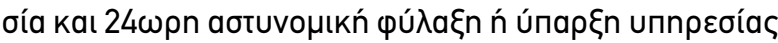

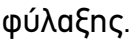

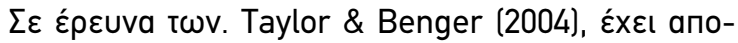

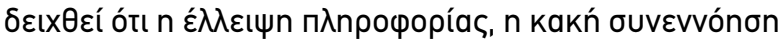

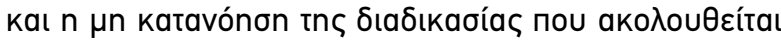

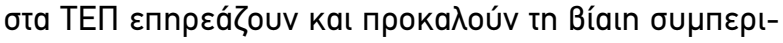

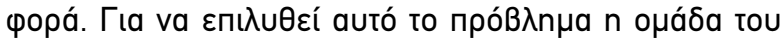
Ballarat Health Service Emergency Department (See

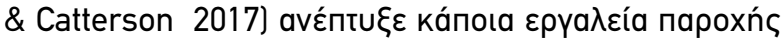

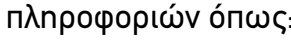

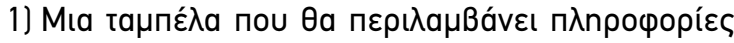

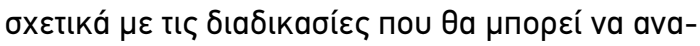

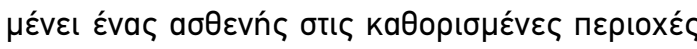

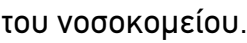

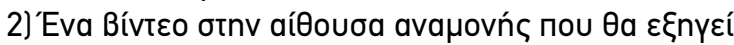

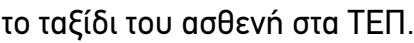

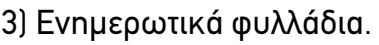

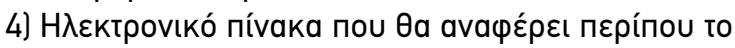

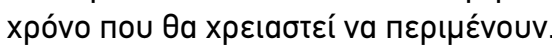

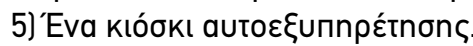

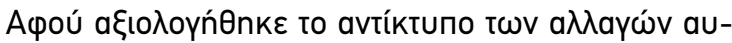

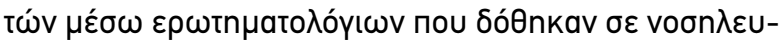

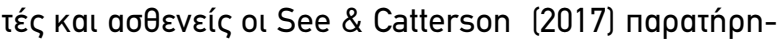

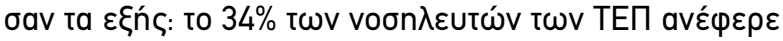

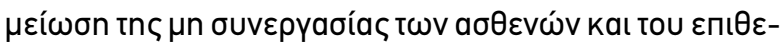

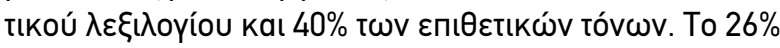

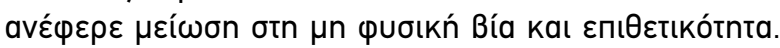

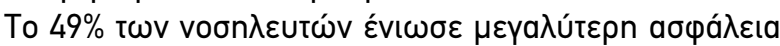

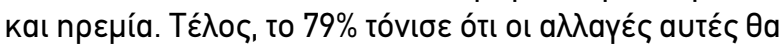
$\varepsilon \Pi n \rho \varepsilon a ́ \sigma o u v$ tnv anópaon tous yıa to av $\theta$ a ouvexíoouv

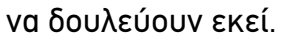

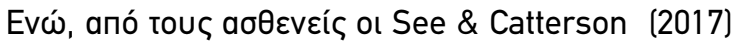

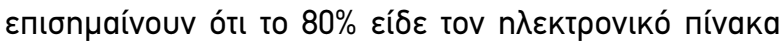

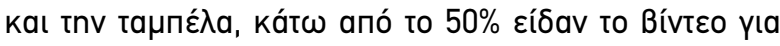

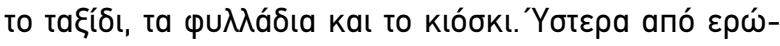

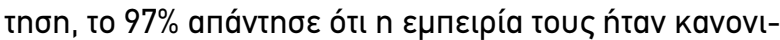

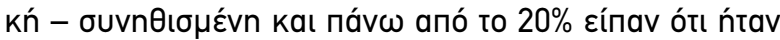

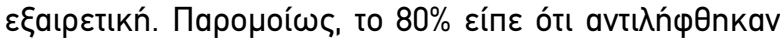

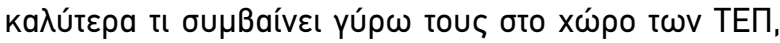

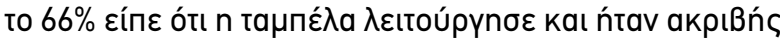

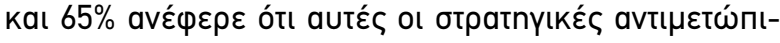

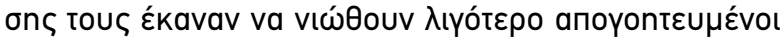

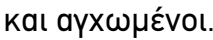

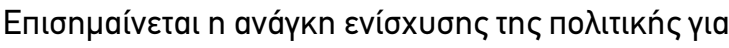

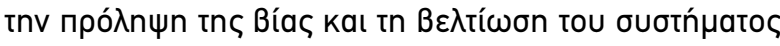

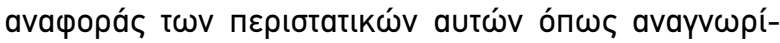

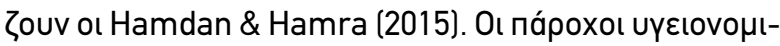

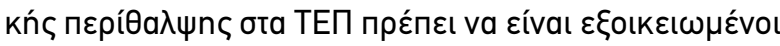

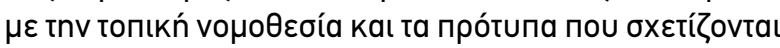

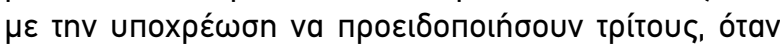

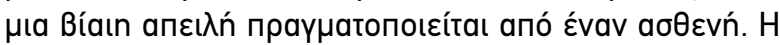

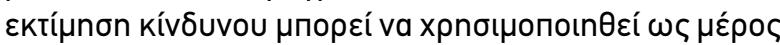

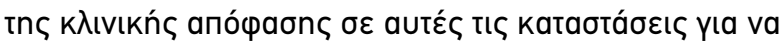

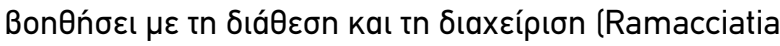
et al 2018).

\section{¿YMПEPAГMATA}

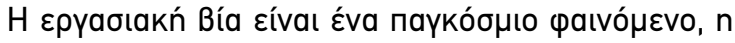

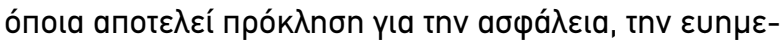

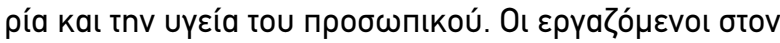

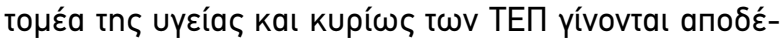

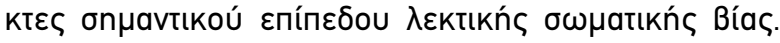

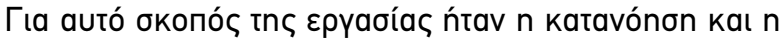

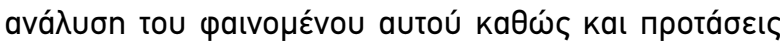

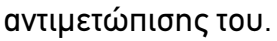

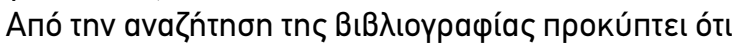

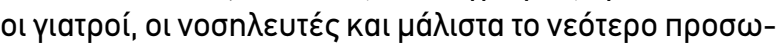

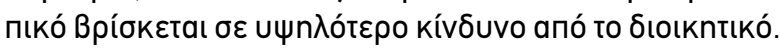

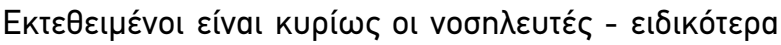

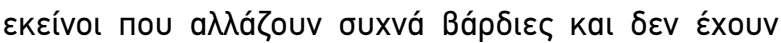

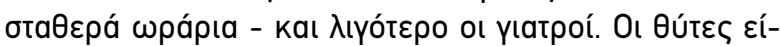

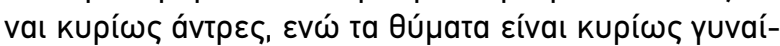

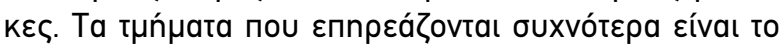

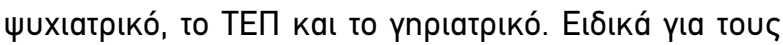

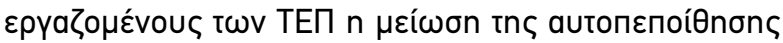

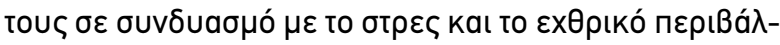

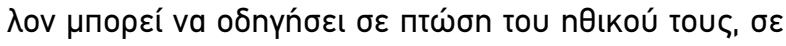




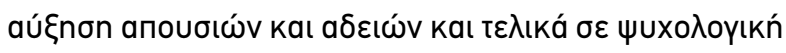

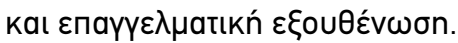

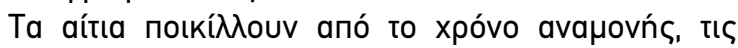

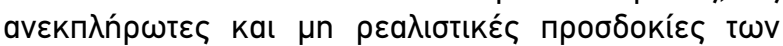

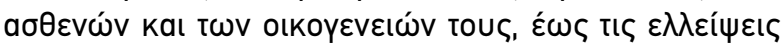

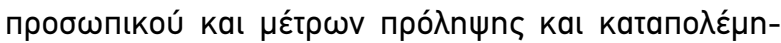

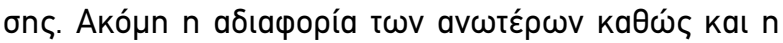

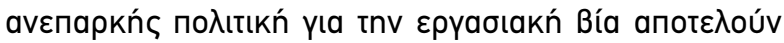

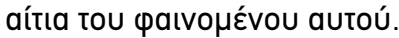

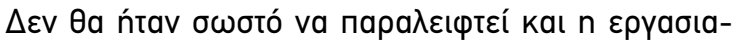

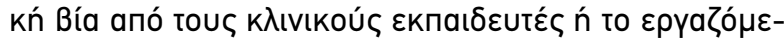

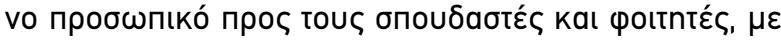

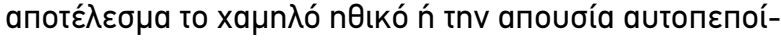

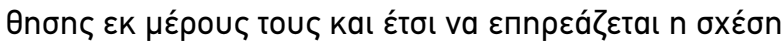

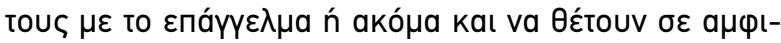

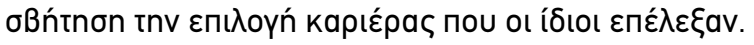

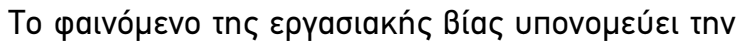

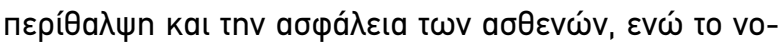

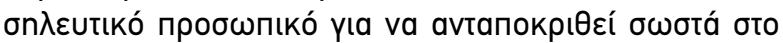

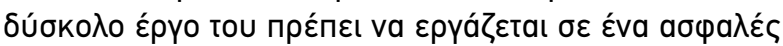

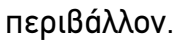

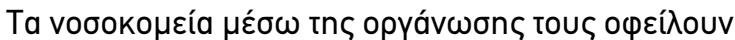

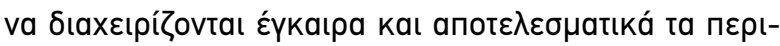

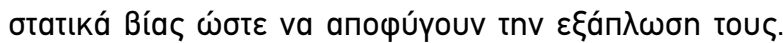

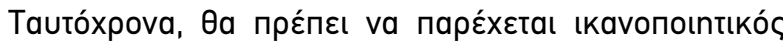

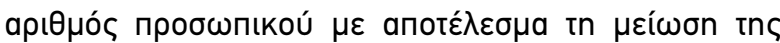

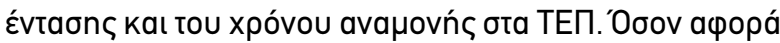

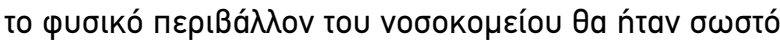

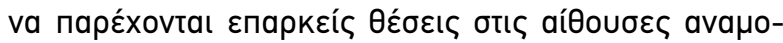

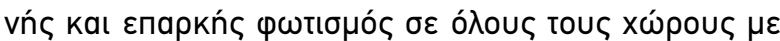

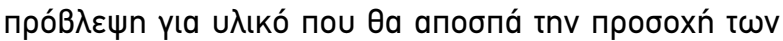

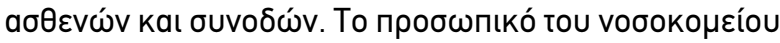

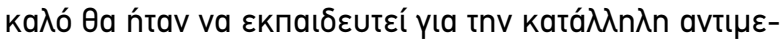

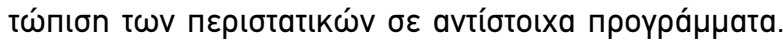

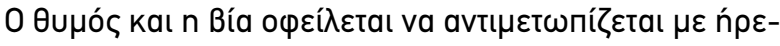

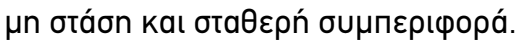

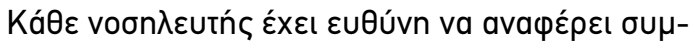

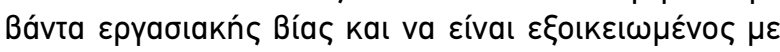

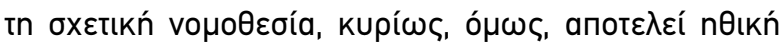

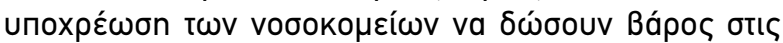

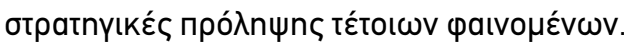




\section{BIB}

American College of Emergency Physicians. (2003). Protection from physical violence in the emergency department. Advanced Emergency Medicine [Online]. Available at: http://newsroom.acep. org/2009-01-04-emergency-department-violence-fact-sheet [Accessed: 21 May 2019].

Baykan Z., Öktem I.S., Çetinkaya F. \& Nacar M. (2015). Physician exposure to violence: a study performed in Turkey. International Journal of Occupational Safety and Ergonomics 21:291-297.

Bayram B., Çetin M., Çolak Oray N. \& Can I.O. (2017). Workplace violence against physicians in Turkey's emergency departments: a cross sectional survey. British Medical Journal Open 7:e013568.

Bowden E. (2017). Nurse taken hostage by knife-wielding patient at Royal Melbourne Hospital. In: The Age [Online]. Available at: https://www.theage.com.au/national/victoria/nurse-takenhostage-by-knifewielding-patient-at-royal-melbourne-hospital20170413-gvk0vq.html [Accessed: 21 May 2019].

Chapman R. \& Styles I. (2006). An epidemic of abuse and violence: Nurse on the front line. Accident and Emergency Nursing 14:245249.

Claravall L. (1996). Healthcare violence: A nursing administration perspective. JONA: The Journal of Nursing Administration 26:4146.

Farrell GA., Bobrowski C. \& Bobrowski P. (2006). Scoping workplace aggression in nursing: Findings from Australian study. Journal of Advanced Nursing 55:778-787.

Fernandes C.M., Bouthillette F., Raboud J.M., Bullock L., Moore C.F, Christenson J.M., Grafstein E., Rae S., Ouellet. L. \& Gillrie C. (1999). Violence in the emergency department: a survey of health care workers. Canadian Medical association Journal 161:12451248.

Ferri P., Silvestri M., Artoni A. \& Di Lorenzo R. (2016). Workplace violence in different settings and among various health professionals in an Italian general hospital: a cross-sectional study. Psychology Research and Behavior Management 23:263275.

Gerberich S.G., Church T.R., McGovern P.M., Hansen H.E., Nachreiner N.M., Geisser M.S., Ryan A.D., Mongin S.J. \& Watt G.D. (2004). An epidemiological study of the magnitude and consequences of work related violence: the Minnesota Nurses' Study. Occupational and Environmental Medicine 61:495-503.

Gillespie G.L., Bresler S., Gates D.M. \& Succop P. (2013). Posttraumatic stress symptomatology among emergency department workers following workplace aggression. Workplace Health Safety 61:247254.

Hakojärvi H., Salminen L. \& Suhonen R. (2012). Health care students personal experiences and coping with bullying in clinical training Nurse Education Today 34:138-144.

Hamdan M. \& Hamra A. (2015). Workplace violence towards workers in the emergency departments of Palestinian hospitals: a crosssectional study. Human Resources for Health 7(13):28-32.

Jiao M., Ning N., Li Y., Lijun G., Yu C., Hong S., Zheng K., Libo L., Qunhong W.V. \& Yanhua H. (2015) Workplace violence against nurses in Chinese hospitals: a cross-sectional survey. British Medical Journal Open 5: e006719.

Krug E.G., Dahlberg L.L., Mercy J.A., Zwi A.B. \& Lozano R. (2002) World report on violence and health. Geneva: World Health Organization [Online]. Available at https://www.who.int/violence_ injury_prevention/violence/world_report/en/ [Accessed: 21 May 2019]
Kwok R., Law Y., Li K., Ng Y., Cheung M., Fung V., Kwok T., Tong J.M., Yen P.F. \& Leung W.C. (2006). Prevalence of workplace violence against nurses in Hong Kong. Hong Kong Medical Journal 12:6-9.

Lanza M., Demaio J. \& Benedict M. (2005). Patient assault support group: achieving educational objectives. Issues in Mental Health Nursing 26:643-660.

Lau J.B., Magarey J. \& Mc Cutcheon H. (2004). Violence in the emergency department: a literature review. Australasian Emergency Nursing Journal 7:27-37.

Lau J.B.C., Magarey J. \& McCutcheon H. (2004). Violence in the emergency department: a literature review. Australian Journal of Emergency Nursing 7:27-37.

Magnavita N. \& Heponiemi T. (2012). Violence towards health care workers in a Public Health Care Facility in Italy: a repeated crosssectional study. BMC Health Services Research 2(12):108-110.

Olding R. (2017). Hospital security crisis: Nurses 'held hostage with meat cleaver' at Wyong. The Sydney Morning Herald [Online]. Available at: https://www.smh.com.au/national/nsw/hospitalsecurity-crisis-nurses-held-hostage-with-meat-cleaver-atwyong-20170410-gvhrtd.html [Accessed: 21 May 2019]

Pich J. \& Kable A. (2014). Patient-related violence against nursing staff working in emergency departments: a systematic review. JBI Database of Systematic Reviews and Implementation Reports 12:398-453

Pich J., Hazelton M., Sundin D. \& Kable A. (2011). Patient-related violence at triage: a qualitative descriptive study. International Emergency Nursing 19:12-19.

Pinar T., Acikel C., Pinar G., Karabulut E., Saggun M., Bariskin E. \& Guidotti T.L. (2015). Workplace violence in the health sector in Turkey: a national study. Journal of Interpersonal Violence 32:2345-2365

Ramacciatia N., Ceccagnolib A., Addeyb B. \& Raseroa L. (2018). Violence towards Emergency Nurses. The Italian National Survey 2016: A qualitative study. International Journal of Nursing Studies 81:21-29.

See J.A. \& Catterson P. (2017) Can improving information to patients about emergency department processes reduce aggressive behavior towards staff? Emergency Medicine Australasia 29:597-606.

Shields M. \& Wilkins K. (2009). Factors related to on-the-job abuse of nurses by patients. Health Reports 20:7-19.

Smith-Pittman M.H. \& Mckoy Y.D. (1999). Workplace violence in healthcare environments. Nursing Forum 34:5-13.

Stathopoulou Ch. (2007). Violence and aggression towards health care professionals. Health Science Journal 1:1-5.

Taylor C. \& Benger J. (2004). Patient satisfaction in emergency medicine. Journal of Emergency Medicine 21:528-352.

Taylor J.L. \& Rew L. (2011) A systematic review of the literature: workplace violence in the emergency department. Journal of Clinical Nursing 20:1072-1085.

Tee S., Üzar Özçetin Y. \& Russell-Westhead M. (2016). Workplace violence experienced by nursing students: A UK survey Nurse Education Today 41:30-35.

Picha J., Kableb A. \& Hazelton M. (2017). paper Antecedents and precipitants of patient-related violence in the emergency department: Results from the Australian VENT Study (Violence in Emergency Nursing and Triage). Australasian Emergency Nursing Journal 20:107-113.

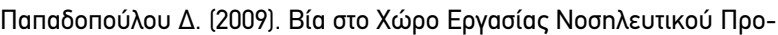

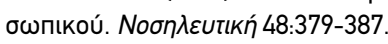




\title{
Violence in the hospital area as a professional scourge in the 21 st century
}

\author{
Konstantinos Gatsios 1 , Martha Stauropoulou-Kelesi², Georgia Fasoi ${ }^{3}$ \\ 1. Student, Faculty of Nursing, University of West Attica \\ 2. Professor, Faculty of Nursing, University of West Attica \\ 3. Assistant Professor, Faculty of Nursing, University of West Attica
}

\section{ABSTRACT}

The phenomenon of violence in the working environment concerns all professions and all countries. Several researchers and studies dealing with violence in the working environment towards health professionals shows the extent of the problem. This phenomenon is more pronounced in the emergency departments (EDs) where violence is an inevitable part of professional life. Healthcare professionals are at higher risk than administrative staff. Especially for ED workers, violence in the working environment can lead to burnout. The causes vary, starting with the excessive waiting time due to lack of staff and ending up in the inadequate policy that most hospitals have for violence in the working environment. The phenomenon of workplace violence undermines the care and safety of patients, while nursing staff in order to fulfill their difficult tasks need a safe environment. Workload, lack of staff and unfulfilled expectations of patients and escorts are not consistent with the proper patients nursing management.

Key words: Aggression in hospitals, emergency departments, health professionals, workplace violence 